\title{
A EPILEPSIA NOS TUMORES CEREBRAIS
}

\author{
LuÍs MARQues-Assis * \\ DANIEL GUENNI-BeJaR ** \\ Evaldo J. FARIA LINS**
}

É admitido que alguns tumores e lesões de certas áreas cerebrais têm maior ou menor poder epileptogênico. Por outro lado, é de observação freqüente o caso de pacientes com tumores cerebrais que em nenhum momento da evolução da doença apresentam manifestações de caráter epiléptico. Embora tais fatos sejam de conhecimento mais ou menos antigo, nem sempre os dados referidos pelos pesquisadores são coincidentes. Assim é que Penfield e col. ${ }^{6}$, em revisão da literatura, verificaram que a incidência da epilepsia nos tumores cerebrais varia de $29 \%$ a $39 \%$. Quanto à localização, Penfield encontrou incidência de $45 \%$ de epilepsia nos processos expansivos supratentoriais, considerada elevada em relação a estudos anteriores referidos na literatura.

No presente trabalho propomo-nos não só a estudar a incidência da epilepsia nos tumores cerebrais pròpriamente ditos, quanto à sua natureza e quanto à sua localização, como também sua relação com o tempo da epilepsia, o tipo da manifestação epiléptica e sua severidade.

\section{MATERIAL, MÉTODOS E RESULTADOS}

De 411 casos de tumores cerebrais internados no Departamento de Neurocirurgia do Hospital das Clínicas de São Paulo, diagnosticados por biópsia ou por necrópsia, foram estudados 86 casos que apresentaram, precedendo a internação, manifestaçōes epilépticas. A idade dos pacientes variou entre 3 e 63 anos; 55 eram do sexo masculino e 31 do sexo feminino; 78 eram brancos, 6 eram pardos e, dois, amarelos. As convulsões surgiram em tempo que oscilou entre um dia e 12 anos antecedendo a data da internação. Em 34 casos as crises eram de tipo grande mal puro e, em 47, eram precedidas por manifestações focais; destas, em 32 casos as manifestações eram de tipo bravais-jacksoniano; em 5 casos as crises eram atípicas. Sinais clínicos de hipertensäo intra-craniana ocorreram em 74 casos, sendo que em 62 havia sinais neurológicos de localização. O exame de liquido cefalorraqueano foi feito em 47 casos, tendo sido normal em 7 , revelando hipertensão em 28 (a pressão foi medida em 43 casos) e com hiperproteinorraquia em 27 (a proteína foi dosada em 45 casos). Em 34 casos foi realizada radiografia simples de crânio, que resultou normal em 11 casos, mostrou sinais de hipertensão intracraniana em 10 casos, calcificações em 6 e erosões ósseas em dois casos. A carotidoangiografia foi feita em 65 casos, tendo sido normal em 4, revelando

Departamento de Neurologia (Prof. Adherbal Tolosa) da Faculdade de Medicina da Universidade de São Paulo: * Assistente; ** Médicos estagiários. 
a presença de tumor hemisférico em 55 e de tumor selar em um caso; em 5 casos êsse exame mostrou sinais indiretos de dilataçãoção ventricular. Os exames neuro-radiológicos contrastados foram completados por pneumencefalografia (10 casos), iodoventriculografia (9 casos) e pneumocisternografia (dois casos).

A epilepsia, quanto à duração, ao tipo de manifestação e à severidade, foi estudada em relação à natureza dos tumores cerebrais e à sua localização.

Os resultados, analisados em indices percentuais, podem ser observados nos quadros 1 a 5 .

\section{COMENTÃ R IOS}

De 411 pacientes com tumores cerebrais diagnosticados por biópsia ou por necrópsia, manifestações epilépticas foram constatadas em 86 casos (19,4\%). Essa incidência è semelhante à verificada por Webster e Weinberger ${ }^{8}$, inferior à de White e col. ${ }^{9}$, diferindo bastante da assinalada por Güvener e col. ${ }^{2}$; enquanto os primeiros encontraram valôres de $21,5 \%$ e de $25,0 \%$ respectivamente, os últimos referiram incidência de 48,1\%; por outro lado, os resultados obtidos por Lund ${ }^{4}(34,1 \%)$ e por Penfield e col. ${ }^{6}$ $(37,0 \%)$ não podem ser comparados com os nossos, pois êsses investigadores incluíram processos expansivos não tumorais em sua casuística.

Nos quadros 1 e 2 pode ser observada a incidência da epilepsia segundo a natureza e segundo a localização dos tumores cerebrais, comparativamente com investigações desenvolvidas por outros autores. Em nossos casos constatamos, conforme era de esperar, que o oligodendroglioma foi o tumor com maior poder epileptogênico $(75 \%)$; também nos carcinomas metastáticos a epilepsia foi freqüentemente registrada (72\%) enquanto que, contràriamente ao que é usualmente referido na literatura, a incidência da epilepsia nos casos com astrocitoma não foi tão elevada $(18 \%)$. Por outro lado, as menores incidências de epilepsia foram encontradas entre os craniofaringeomas $(10 \%)$, os tumores de hipófise $(0 \%)$ e os pinealomas $(0 \%)$. Quanto à topografia dos tumores cerebrais que mais freqüentemente era acompanhada de manifestações epilépticas, verificamos concordância no que se refere à alta incidência das crises nas neoplasias das regiões frontais $(39 \%)$, parietais $(38 \%)$ e temporais $(31 \%)$, assim como à baixa incidência nos tumores da fossa posterior (11\%), da sela túrsica (5\%) e da região da pineal $(0 \%)$.

No quadro 3 procuramos relacionar o tempo de duração da epilepsia com a natureza e com a topografia dos tumores. Verifica-se, quanto à natureza, que entre os casos com início tardio da epilepsia, o carcinoma metastático apareceu como o tumor mais freqüente; nos casos de evolução mais longa, o astrocitoma, em primeiro lugar e o meningeoma, em segundo lugar, seguido do glioblastoma multiforme, prevaleceram dentre os demais tipos de tumores. Penfield e col. ${ }^{6}$, estudando o tempo de evolução da epilepsia em pacientes com tumor cerebral, verificaram maior possibilidade de longa evolução nos astrocitomas $(75 \%)$ que nos glioblastomas $(25 \%)$. O mesmo fato foi ressaltado por Lund ${ }^{4}$, que assinalou a probabilidade de $2 \%$ dos glioblastomas produzirem epilepsia com evolução superior a dois anos, contra $14 \%$ para os meningeomas e $52 \%$ para outros gliomas. A 
Natureza do tumor

List

(1936)

Penfield e col.

(1940)

White e col.

(1948)

Güvener e col.

Marques-Assis

(1964)

e col. (1969)

Oligodendrogliomas

(33) $-25-76 \%$

(13) $-?-92 \%$

(19) $-7-37 \%$

(4) $-3-75 \%$

Metástases carcinomatosas

$$
\text { (35) }-5-14 \%
$$

(11) $-8-72 \%$

\section{Meningeomas}

Glioblastomas multiformes

(125) $-49-39 \%$

(63) $-?-67 \%$

(99) $-35-35 \%$

(?) - ? - 53\%

(40) $-18-45 \%$

Ependimomas

(17) $-4-23 \%$

(103) - ? - $37 \%$

(145) $-32-22 \%$

(?) $-?-35 \%$

(85) $-19-22 \%$

(9) $-?-50 \%$

(33) $-11-33 \%$

(24) $-5-20 \%$

Tumores angiomatosos

\section{Meduloblastomas}

Astrocitomas

(113) $-74-65 \%$

$(64-?-70 \%$

(88) $-44-50 \%$

(21) $-4-20 \%$

(32) $-6-19 \%$

(62) $-11-18 \%$

Craniofaringeomas

(?) $-0-0 \%$

(33) $-3-10 \%$

Tumores de hipófise

(?) - ? - $12 \%$

(39) $-0-0 \%$

Pinealomas

(12) $-0-0 \%$

QUADRO 1 - Incidência da epilepsia segundo a natureza dos tumores mais freqüentes, comparativamente a investigaçóes de-

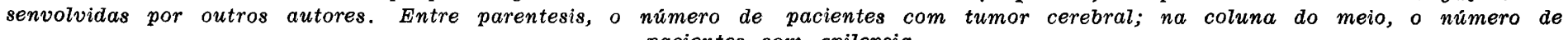
pacientes com epilepsia. 


\begin{tabular}{|c|c|c|c|c|}
\hline Localização do tumor & $\begin{array}{l}\text { Penfield e col. } \\
\qquad(1940)\end{array}$ & $\begin{array}{l}\text { White e col. } \\
\text { (1948) }\end{array}$ & $\begin{array}{l}\text { Lund } \\
\text { (1952 }\end{array}$ & $\begin{array}{c}\text { Marques-Assis e col. } \\
\text { (1969) }\end{array}$ \\
\hline
\end{tabular}

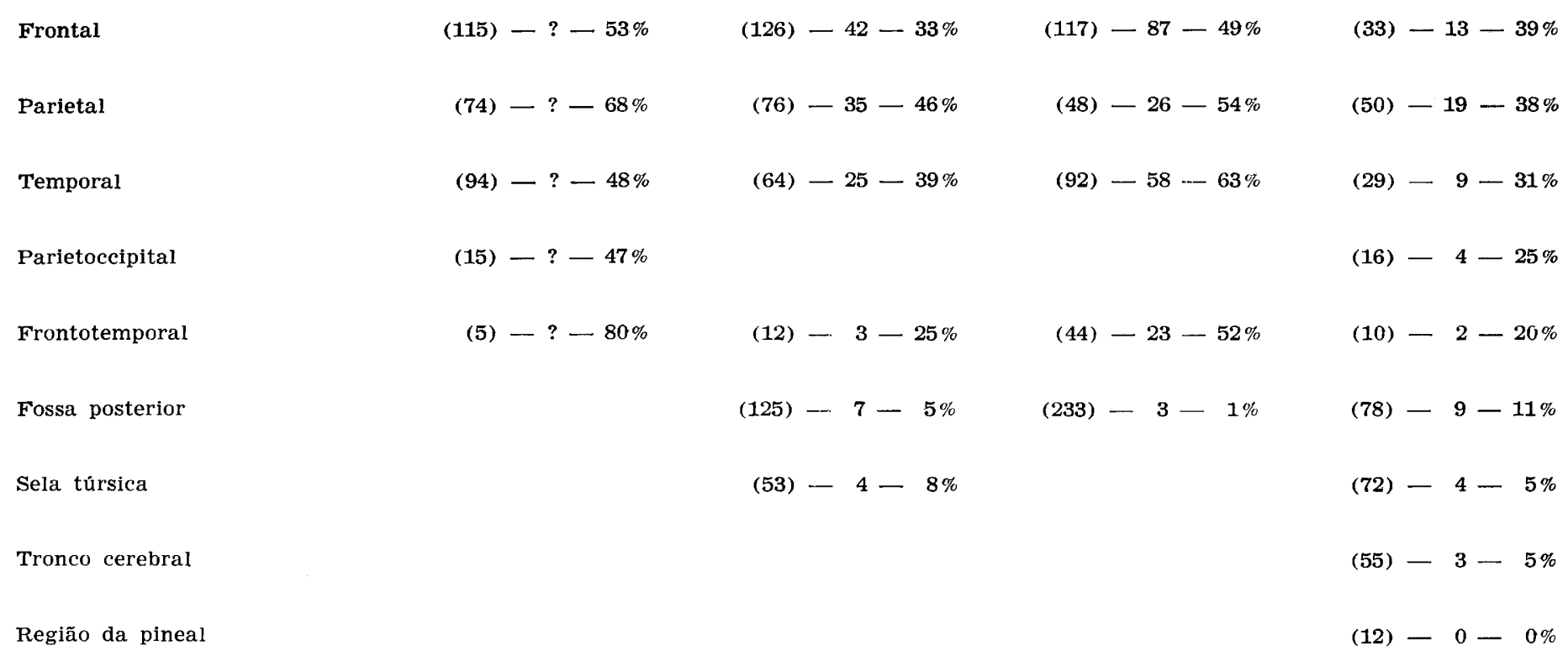

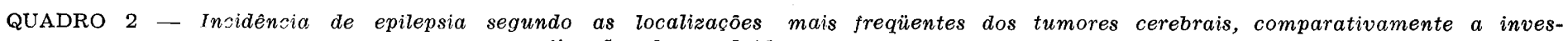
tigacões desenvolvidas por outros autores. 


\begin{tabular}{|c|c|c|c|c|}
\hline Duraçāo da epilepsia & Natureza do tu & & Localização & do tumor \\
\hline Inferior a um ano & $\begin{array}{l}\text { Carcinoma metastático } \\
\text { Meningeoma } \\
\text { Ependimoma }\end{array}$ & $\begin{array}{l}(11)-4-36 \% \\
(40)-7-17 \% \\
(24)-4-16 \%\end{array}$ & $\begin{array}{l}\text { Frontal } \\
\text { Parietoccipital } \\
\text { Temporal }\end{array}$ & $\begin{array}{l}(33)-6-18 \% \\
(16)-3-18 \% \\
(29)-5-17 \%\end{array}$ \\
\hline De 1 a 3 anos & $\begin{array}{l}\text { Astrocitoma } \\
\text { Meningeoma } \\
\text { Glioblastoma multiforme }\end{array}$ & $\begin{array}{l}(11)-6-54 \% \\
(18)-8-44 \% \\
(19)-6-31 \%\end{array}$ & $\begin{array}{l}\text { Foice do cérebro } \\
\text { Parietal } \\
\text { Temporal }\end{array}$ & $\begin{array}{r}\text { (3)-2-66\% } \\
(19)-9-47 \% \\
(9)-4-44 \%\end{array}$ \\
\hline Superior a três anos & $\begin{array}{l}\text { Astrocitoma } \\
\text { Meningeoma } \\
\text { Glioblastoma multiforme }\end{array}$ & $\begin{array}{l}(11)-5-45 \% \\
(18)-4-22 \% \\
(19)-2-10 \%\end{array}$ & $\begin{array}{l}\text { Temporal } \\
\text { Frontal } \\
\text { Parietal }\end{array}$ & $\begin{array}{l}(9)-4-44 \% \\
(13)-3-23 \% \\
(19)-4-21 \%\end{array}$ \\
\hline
\end{tabular}

QUADRO 3 - Relação entre a duração da epilepsia e a natureza e a localização do tumor.

Freqüência diária até quinzenal das crises

\begin{tabular}{|c|c|c|}
\hline Natureza do & tumor & Localização do tumor \\
\hline Oligodendroglioma. & (4) $-2-50 \%$ & Frontotemporal (2) $-1-50 \%$ \\
\hline Meningeoma & $(18)-4-22 \%$ & (3) $-1-33 \%$ \\
\hline Carcinoma metastático & $(8)-1-12 \%$ & $(19)-5-26 \%$ \\
\hline Glioblastoma multiforme & $(19)-2-10 \%$ & (5) $-1-20 \%$ \\
\hline Astrocitoma & (11) $-1-9 \%$ & $(13)-2-15 \%$ \\
\hline
\end{tabular}




\begin{tabular}{|c|c|c|c|c|c|c|c|}
\hline \multirow{3}{*}{ Crises GM puras } & Craniofaringeoma & (3) & $-3-100 \%$ & Sela túrsica & (4) & $-4-$ & $100 \%$ \\
\hline & Meduloblastoma & (6) & $-4-$ & Fossa posterior & (9) & $-6-$ & $66 \%$ \\
\hline & Glioblastoma multiforme & (19) & $-9-47 \%$ & Temporal & (9) & $-4-$ & $44 \%$ \\
\hline \multirow{3}{*}{ Convulsões focais } & Oligodendroglioma & (4) & $-2-50 \%$ & Foice do cérebro & (3) & $-2-$ & $66 \%$ \\
\hline & Glioblastoma multiforme & (19) & $-4-$ & Frontal & (13) & $-3-$ & $23 \%$ \\
\hline & Astrocitoma & (11) & $-2-$ & Temporal & (9) & $-2-$ & $22 \%$ \\
\hline \multirow{3}{*}{ Crises bravais-jacksonianas } & Carcinoma metastático & (8) & $-4-50 \%$ & Frontotemporal & (5) & $-4-$ & $80 \%$ \\
\hline & Meningeoma & (18) & $-9-$ & Parietal & (19) & $-8-$ & $42 \%$ \\
\hline & Glioblastoma multiforme & (19) & $-5-26 \%$ & Frontal & (13) & $-4-$ & $30 \%$ \\
\hline
\end{tabular}

QUADRO 5 - Relação entre as características da crise epiléptica e a natureza e a localização do tumor. 
êsse propósito List $^{3}$ fêz estudo mais completo; segundo o autor, dentre os gliomas o oligodendroglioma, em primeiro lugar, e o astrocitoma, em segundo lugar, são aquêles que mais fàcilmente podem determinar o aparecimento de epilepsia de longa evolução, tanto assim que em 14\% dos casos de oligodendroglioma e em $9 \%$ dos casos de astrocitoma, a evolução ocorreu em tempo superior a 10 anos. Paillas e col. 5 estudando 34 casos com oligodendroglioma, encontraram a epilepsia como primeira manifestação da moléstia em 18 casos $(52,9 \%)$. Quanto à topografia das neoplasias encefálicas, em nosso material os casos com epilepsia de evolução mais longa apresentaram localização preferentemente temporal (44\%), enquanto que os de evolução mais rápida predominaram nas regiões frontal (18\%) e parietoccipital $(18 \%)$.

Lund ${ }^{4}$, estudando a natureza dos tumores cerebrais em relação à freqüência das crises epilépticas verificou que, nas formas mais severas, com freqüência superior a uma crise por semana, predominaram nitidamente os gliomas, seguindo-se os meningeomas e os glioblastomas. Em nossos casos (quadro 4), nas formas mais severas da epilepsia, o oligodendroglioma $(50 \%)$, o meningeoma $(22 \%)$ e o carcinoma metastático $(12 \%)$ foram os tumores mais freqüentemente encontrados. Quanto à topografia (quadro 4), no mesmo grupo de casos, predominaram as regiões fronto-temporais $(50 \%)$, a foice do cérebro $(33 \%)$ e as regióes parietais $(26 \%)$.

No que se refere às manifestações convulsivas puras relativamente à natureza e à localização dos tumores cerebrais (quadro 5) nota-se, como era de se esperar, predominância dos craniofaringeomas e dos meduloblastomas, quanto à natureza, e da sela túrsica e fossa posterior, quanto à topografia. Penfield e Jasper ${ }^{7}$ observaram não haver evidências clínicas de que lesões cerebelares modifiquem ou produzam aumento da tendência a crises; Penfield e col. ${ }^{6}$, em trabalho anterior, dão incidência de epilepsia de $20 \%$ nos tumores infratentoriais, embora chamando a atenção para o fato de que raramente as crises assumem aspecto convulsivo típico. Webster e Weinberger ${ }^{8}$, estudando especìficamente a incidência da epilepsia em 158 pacientes com tumores intracerebelares, encontraram manifestações comiciais em $21,5 \%$ dos casos; os autores concluíram que a existência de crises convulsivas focais ou generalizadas não deve excluir a possibilidade de sede cerebelar para a lesão. White e col. ${ }^{9}$ observaram a incidência de epilepsia em 5\% dos casos com tumor infratentorial. Em nossos casos (quadros 2 e 5) os tumores da fossa posterior (exceto tronco cerebral) determinaram o aparecimento de epilepsia em $11 \%$ dos casos; por outro lado os tumores de cerebelo, isoladamente considerados, desenvolveram atividade epileptogênica em 8 casos $(10,2 \%)$. No que se refere às convulsões focais (quadro 5), observamos predominância nítida dos oligodendrogliomas quanto à natureza e da foice do cérebro quanto à localização. Gibbs ${ }^{1}$, estudando 1.545 casos de tumor cerebral, chamou a atenção para a alta incidência de crises focais nas localizacões parietais. 
List ${ }^{3}$, investigando 300 casos de gliomas de hemisférios cerebrais, ressaltou como localizações principais para as manifestações bravais-jacksonianas as regiōes frontoparietal $(50 \%)$, parietal $(43 \%)$ e frontal $(33 \%)$. Em nosso trabalho (quadro 5) encontramos resultados semelhantes, com predominância das regiões frontoparietal (80\%), parietal $(42 \%)$ e frontal $(30 \%)$. Quanto à natureza do tumor (quadro 5) os carcinomas metastáticos e os meningeomas foram aquêles que mais freqüentemente determinaram o aparecimento de crises bravais-jacksonianas.

\section{RESUMO E CONCLUSÓES}

De 411 casos de tumores cerebrais foram estudados $86(19,4 \%)$ que apresentaram manifestações epilépticas. A epilepsia foi estudada quanto à duração, ao tipo de manifestação e à severidade, em relação à natureza e à localização dos tumores cerebrais.

A análise dos resultados permitiu aos autores chegarem às seguintes conclusões: 1) a epilepsia incidiu em 19,4\% dos casos; 2) o oligodendroglioma, as metástases carcinomatosas e o glioblastoma multiforme foram, pela ordem, os tumores mais epileptogênicos; 3 ) as áreas frontal, parietal e temporal foram, nessa ordem, as localizações mais epileptogênicas; 4) os carcinomas metastáticos predominaram nos casos com epilepsia com menos de um ano de evolução, enquanto que os astrocitomas predominaram nos casos com mais de três anos; quanto à topografia, predominou a região frontal no primeiro grupo e a temporal, no segundo; 5) nas formas mais severas de epilepsia predominaram os oligodendrogliomas e os meningeomas, quanto à natureza, e a região frontotemporal e a foice, quanto à localização; 6) os craniofaringeomas e os meduloblastomas foram os tumores que mais freqüentemente determinaram o aparecimento de convulsões puras; nesses casos, a sela túrsica e a fossa posterior foram as sedes mais freqüentes; 7) os carcinomas metastáticos e os meningeomas, quanto à natureza, e as regiões frontoparietal e parietal, quanto à localização, foram os mais freqüentemente encontrados nos casos com crises de tipo bravais-jacksoniano.

\section{S U M M A R Y}

\section{Epilepsy in cerebral tumors}

In 411 cases of cerebral tumors, 86 (19,4\%) with epileptic manifestations were studied. The duration, the manifestations and the severity of eplepsy, in regard to the nature and site of the tumor, were investigated. 
The analysis of the results lead the authors to the following conclusions: 1) $19.4 \%$ of the cerebral tumors showed epileptic symptoms; 2) oligodendroglioma, metastatic carcinomas and glioblastoma multiforme were, in this order, the most epileptogenic tumors; 3 ) the frontal, parietal and temporal areas were, in this order, the most epileptogenic localizations; 4) concerning the duration of epilepsy, the metastatic carcinomas were more frequent when the duration of epilepsy was lower than one year, while the astrocytoma were more frequent in the cases with more than three years; regarding the location, the frontal area was more frequent in the first group and the temporal area in the second one; 5) regarding the frequency of seizures, oligodendroglioma and meningeoma, and the frontotemporal and falx cerebri locations were found more frequently in the more severe cases; 6) concerning the pattern of epileptic manifestations, cranio-pharyngiomas and meduloblastomas were the tumors that induced most seizure without aura; in these cases the pituitary region and the posterior fossa were the more frequent sites; 7) metastatic carcinomas and meningeomas, as well as frontoparietal and parietal areas, were most frequently found in the cases with jacksonian seizures.

\section{R E F E R H C I A S}

1. GIBBS, F. A. - Frequency with which tumors in various parts of the brain produce certain symptoms. Arch. Neurol. Psychit. (Chicago) 28:969989, 1931.

2. GÜVEnER, A.; BAGGHI, B. K.; KOOI, K. A. \& CAlhoun, H. D. Mental and seizure manifestations in relation to brain tumors. A statistical study. Epilepsia 5:166-176, 1964.

3. LIST, C. F. - Epileptiform attacks in cases of glioma of the cerebral hemispheres. Relation to the location and histologic type of the glioma. Arch. Neurol. Psychiat. (Chicago) 35:323-350, 1936.

4. LUND, M. - Epilepsy in association with intracranial tumor. Acta psychiat. neurol. scand. supl. 81, 1952.

5. PAILlAS, J. E.; COMBALBERT, A.; BERARD-BADER, M.; BILLE, J. \& FRANK, R. - Etude sur l'évolution de oligodendrogliomes de l'encéphale. A propos d'une série opératoire de 34 cas. Acta neurol. psychiat. belg. 64: 537-551, 1964.

6. PENFIELD, W.; ERICKSON, T. C. \& TARLOV, I. - Relation of intracranial tumors and symptomatic epilepsy. Arch. Neurol. Psychiat. (Chicago) $44: 300-315,1940$.

7. PENFIEL, W.; JASPER, H. - Epilepsy and the Functional Anatomy of the Human Brain. Little-Brown Co., Boston, 1954. 
8. WEBSTER, J. E. \& WEINBERGER, L. M. - Convulsions associated with tumors of the cerebelum. Clinical and pathophysiologic features. Arch. Neurol. Psychiat. (Chicago) 43:1163-1184, 1940.

9. WHITE, J. C.; LIU, C. T. \& MIXTER, W. J. - Focal epilepsy. A statistical study of its causes and the results of surgical treatment. I: Epilepsy secondary to intracranial tumors. New Engl. J. Med. 238:891-899, 1948.

Departamento de Neurologia - Faculdade de Medicina da Universidade de São Paulo - Caixa Postal $\$ 461$ - São Paulo, SP - Brasil. 